\title{
Warum malte Adam Elsheimer den Mond auf dem Kopf? - Teleskopisches Sehen ist ohne Teleskop möglich
}

\section{Why Did Adam Elsheimer Paint the Moon Upside-Down? Telescopic Vision Is Possible Without a Telescope}

\section{Einleitung \\ $\nabla$}

In den letzten Jahren erschienen viele hochwertige Publikationen $\mathrm{zu}$ dem Kunstmaler Adam Elsheimer, der 1610 mit nur 32 Jahren in Rom starb. Mehrere dieser Bücher [1-3] hatten auf der Umschlagseite das Bild „Die Flucht nach Ägypten“, das in der alten Pinakothek in München ausgestellt ist ( $\bullet$ Abb. 1).
Das bekannte Werk enthält Details von Mond und Milchstraße, die nur teleskopisch zu erkennen sind. Aber es entstand vor der Einführung von Fernrohren in die Astronomie! Wie konnte Elsheimer teleskopisch sehen und warum malte er den Mond auf dem Kopf? Eine schlüssige Erklärung dafür steht noch aus. Im Folgenden wird ein Lehrsatz von Johannes Kepler aus dem Jahr 1611 aufgegriffen und weiterentwickelt, der zu einer einfachen
Lösung des Problems führt und zeigt, dass auch heute ein junger Mensch ohne Teleskop, nur durch eine schwache Linse, Mond und Sterne mühelos bis zu 10-fach vergrößert sehen kann.

\section{Das Teleskop \\ $\nabla$}

Ein Linsenfernrohr besteht aus 2 optischen Bauteilen: dem Okular, das dem Auge zugewandt ist und dem Objektiv am anderen Ende des Rohres. Anfänglich bestanden beide aus je 1 Linse. Einfache Amateurinstrumente haben heute mindestens 2 Linsen im Objektiv und mindestens 3 im Okular, bessere Okulare haben bis zu 9 Linsen, um bei guter Qualität hohe Vergrößerungen und große Gesichtsfelder zu erhalten.

Die erste schriftliche Bezeugung eines optisch leistungsfähigen Teleskops gibt es vom 2. Oktober 1608, als der 38-jährige Optiker Hans Lippershey (1570-1619) ein Patent darauf beantragte. Das Gesuch

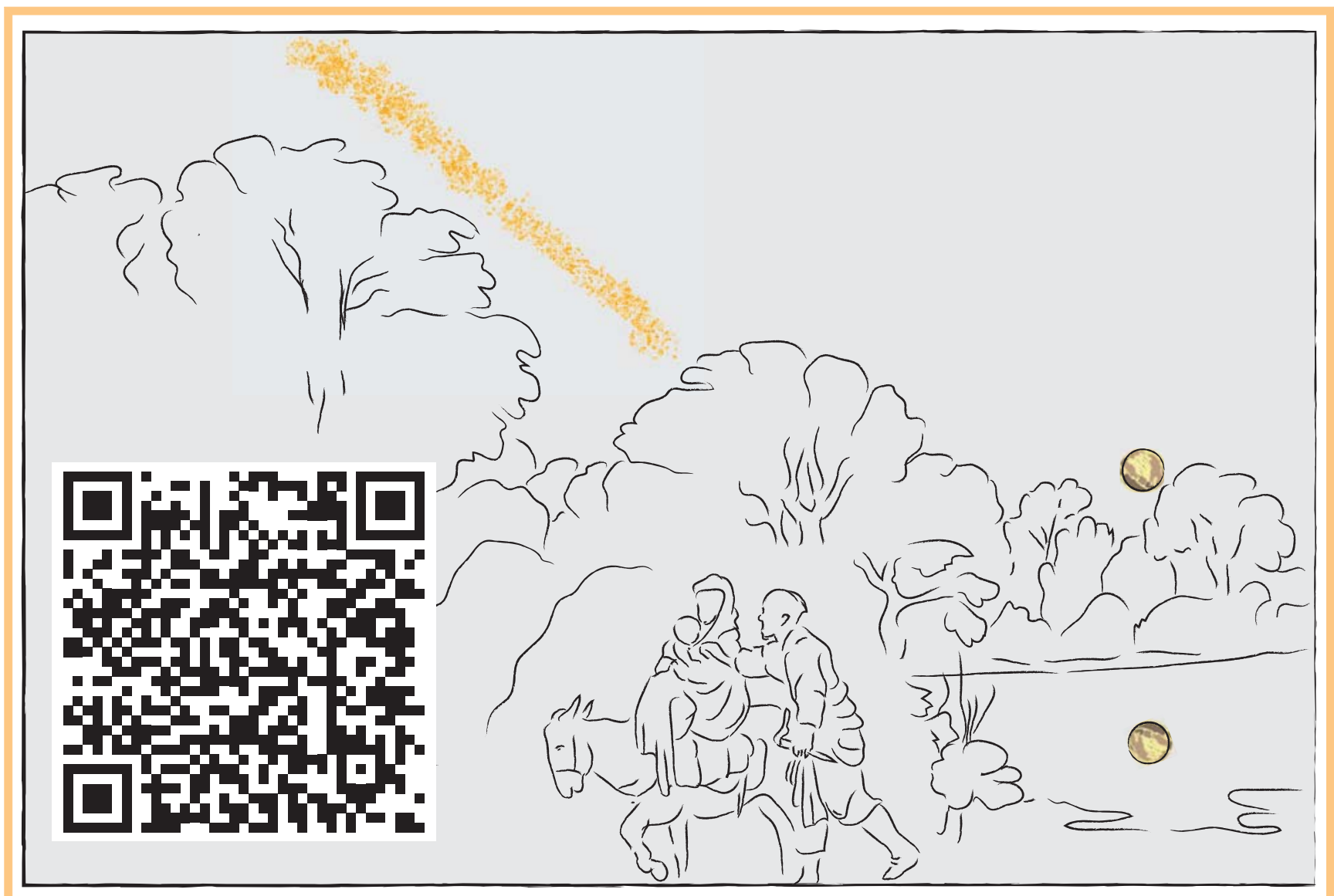

Abb. 1 Flucht (der Jungfrau) nach Ägypten. Adam Elsheimer, ca. 1608. Erste Darstellung der Milchstraße aus Einzelsternen und eines vergrößerten Bildes vom Mond. Bayerische Staatsgemäldesammlungen, bpk (Alte Pinako- thek, München). Im Internet: www.pinakothek.de/adam-elsheimer/dieflucht-nach-aegypten 
Tab. 1 Abbildung von Teleskopen in Abhängigkeit von den Brennweiten.

\begin{tabular}{lllll} 
Brennweite Objektivlinse & Brennweite Okularlinse & Länge & Vergrößerung & Abbildung \\
$+1 \mathrm{dpt}, 100 \mathrm{~cm}$ & $+10 \mathrm{dpt}(10 \mathrm{~cm})$ & $110 \mathrm{~cm}$ & 10 -fach & $\begin{array}{l}\text { auf dem Kopf } \\
\text { + } 1 \mathrm{dpt}, 100 \mathrm{~cm}\end{array}$ \\
$+5 \mathrm{dpt}(20 \mathrm{~cm})$ & $+120 \mathrm{~cm}$ & 5 -fach & auf dem Kopf \\
\hline$+2 \mathrm{dpt}, 50 \mathrm{~cm}$ & $+5 \mathrm{dpt}(10 \mathrm{~cm})$ & $70 \mathrm{~cm}$ & 5 -fach & auf dem Kopf \\
\hline$+2 \mathrm{dpt}, 50 \mathrm{~cm}$ & $-10 \mathrm{dpt}(10 \mathrm{~cm})$ & $90 \mathrm{~cm}$ & 10 -fach & richtig herum \\
\hline$+1 \mathrm{dpt}, 100 \mathrm{~cm}$ & $-5 \mathrm{dpt}(20 \mathrm{~cm})$ & $80 \mathrm{~cm}$ & 5 -fach & richtig herum \\
\hline$+1 \mathrm{dpt}, 100 \mathrm{~cm}$ & $-10 \mathrm{dpt}(10 \mathrm{~cm})$ & $40 \mathrm{~cm}$ & 5 -fach & richtig herum \\
\hline$+2 \mathrm{dpt}, 50 \mathrm{~cm}$ & $-5 \mathrm{dpt}(20 \mathrm{~cm})$ & $30 \mathrm{~cm}$ & 2,5 -fach & richtig herum \\
\hline$+2 \mathrm{dpt}, 50 \mathrm{~cm}$ & & &
\end{tabular}

Tab. 2 Vergrößerungen, die durch Akkommodation, Myopie oder Hyperopie erzielt werden.

\begin{tabular}{|c|c|c|c|c|c|}
\hline $\begin{array}{l}\text { Brennweite Linse } \\
\text { (Objektiv) }\end{array}$ & $\begin{array}{l}\text { Akkommodation } \\
+3 \mathrm{dpt}\end{array}$ & $\begin{array}{l}\text { Akkommodation } \\
+5 \mathrm{dpt}\end{array}$ & $\begin{array}{l}\text { Akkommodation } \\
+10 \mathrm{dpt}\end{array}$ & $\begin{array}{l}\text { Fehlsichtigkeit } \\
\pm 3 \mathrm{dpt}\end{array}$ & $\begin{array}{l}\text { Fehlsichtigkeit } \\
\pm 5 \mathrm{dpt}\end{array}$ \\
\hline$+1 \mathrm{dpt}(100 \mathrm{~cm})$ & 3-fach & 5-fach & 10-fach & 3-fach & 5 -fach \\
\hline$+1,5 \mathrm{dpt}(67 \mathrm{~cm})$ & 2-fach & 3,3-fach & 6,7-fach & 2-fach & 3,3-fach \\
\hline$+2 \mathrm{dpt}(50 \mathrm{~cm})$ & 1,5 -fach & 2,5-fach & 5 -fach & 1,5 -fach & 2,5-fach \\
\hline
\end{tabular}

wurde abgelehnt, weil das optische Prinzip so einfach war, dass es damals schon Nachbauten gab $[4,5]$. Lippershey hat für sein Fernrohr als Objektiv das Glas einer schwachen Lesebrille von etwa $+1,5 \mathrm{dpt}$ und einem konkaven Myopiebrillenglas von etwa - $8 \mathrm{dpt}$ als Okular in einer Metallröhre befestigt. Die Erfindung entstand durch Probieren, ohne Berechnungen [6]. Die Erfindung des astronomischen Fernrohrs mit einer oder mehreren konvexen Okularlinsen beruht auf Untersuchungen, Berechnungen und Überlegungen von Johannes Kepler 1611 [7]. Einer der wenigen, der Keplers komplizierte Rechnungen verstanden hat und derartige Teleskope herstellen konnte, war der Jesuit Christoph Scheiner 1613. Scheiner war es auch, der die Richtigkeit der neuen Theorie Keplers durch Experimente und Sektionen von Augen bestätigte, weiterentwickelte und als Erster die Akkommodation als eine Änderung des Krümmungsradius der Linse richtig erkannte [4,8]. Den Namen „Teleskop“ führte Federico Cesi (1585-1630) in Rom ein, nachdem er 1609 ein Teleskop nach Giambattista della Porta (1535-1615) erhalten hatte ([3], S. 146-147).

Zu Beginn des 17. Jahrhunderts gab es einen sehr großen Markt für Lesebrillen. Die Brillenstärke lag damals wie heute meist zwischen +1 und +4 dpt. Die Gläser waren flach, oft sphärisch plankonvex. Konkave Linsen zur Behandlung der Myopie gab es nur in sehr geringen Stückzahlen. Willach schätzt, dass der Anteil der Myopiebrillen an allen Brillen damals unter 1\% lag [5]. Die Qualität der Brillengläser zu dieser Zeit war sehr un- terschiedlich und nur wenige waren so gut, dass sie sich für Teleskope eigneten. Für astronomische Fernrohre wurden die Linsen mit einer konzentrischen Blende abgedeckt, da bei höheren Vergrößerungen Unregelmäßigkeiten am Rand die Abbildungsqualität dramatisch reduzieren [5].

In der Astronomie werden Linsen durch ihre Brennweite bestimmt, in der Augenheilkunde und Optik durch den Kehrwert der Brennweite, der Dioptrie (dpt). Eine Linse mit einer Brennweite von $100 \mathrm{~cm}$ hat den Wert $+1 \mathrm{dpt}$, eine Brennweite von $50 \mathrm{~cm}$ entspricht $+2 \mathrm{dpt}$ ( Tab. 1 und 2). Um mit einer einfachen Objektivlinse eine einigermaßen scharfe Abbildung zu bekommen, muss die Brennweite groß sein. Die ersten Fernrohrobjektive zu Beginn des 17. Jahrhunderts hatten Brennweiten von etwa 60 bis $130 \mathrm{~cm}$, ihr Dioptrienwert lag zwischen $+1,7$ und + 0,8 dpt ([4], S. 44). Je geringer der Dioptrienwert des Objektivs und je länger die Brennweite wird, um so höhere Vergrößerungen sind möglich. Um die Saturnringe in der 2. Hälfte des 17. Jahrhunderts erkennen zu können, benutzte Christian Huygens 1656 ein $7 \mathrm{~m}$ langes Teleskop $(+0,14 \mathrm{dpt})$ und später mit Giovanni Cassini bis zu $40 \mathrm{~m}$ lange Teleskope $(+0,025 \mathrm{dpt})$. Dazu benutzten sie auch „Luftfernrohre“, getrennt fixierte Okulare und Objektive ohne ein verbindendes Rohr. Dieser Name zeigt die Schwäche des deutschen Wortes, die für das Verstehen des Weiteren wichtig ist: Das „Rohr“ ist ein vernachlässigbares Teil eines Teleskops, wichtig sind die beiden Linsensysteme ([4], S. 73-76). Mit der Etablierung der Spiegelteleskope im 17. Jahrhundert und der Entwicklung der 2-linsigen Linsenobjektive (Achromate) im 18. Jahrhundert konnten hohe Vergrößerungen mit kürzeren Instrumenten verwirklicht werden [4], S. 87-115).

Es ist eher unwesentlich, ob das Okular eine konkave oder eine konvexe Linse ist. Bei einer konkaven, also einer Minuslinse, ist das Bild im Teleskop richtig herum abgebildet und die optische Länge des Fernrohrs errechnet sich aus der Brennweite des Objektivs minus der Brennweite des Okulars. Bei einer konvexen, also einer Pluslinse, ist das Bild im Teleskop höhenund seitenverkehrt, „auf dem Kopf stehend" abgebildet und die optische Länge des Fernrohrs entspricht der Brennweite des Objektivs plus der Brennweite des Okulars ( Tab. 1).

Bei einem Objektiv mit einer + 1-dpt-Linse (Brennweite $100 \mathrm{~cm}$ ) und einem Okular von $-5 \mathrm{dpt}$ (Brennweite $20 \mathrm{~cm}$ ) beträgt die Vergrößerung 5-fach und das Fernrohr ist $80 \mathrm{~cm}$ lang. Bei einem Objektiv mit einer + 1-dpt-Linse (Brennweite $100 \mathrm{~cm}$ ) und einem Okular von $+5 \mathrm{dpt}$ (Brennweite $20 \mathrm{~cm}$ ) beträgt die Vergrößerung ebenfalls 5-fach und das Fernrohr ist $120 \mathrm{~cm}$ lang ( Tab. 1). Um eine Vergrößerung zu erzielen, muss das Okular eine Brennweite haben, die größer als die des Objektivs ist. Die Vergrößerung lässt sich durch das Verhältnis der beiden Brennweiten berechnen ( Tab. 1). Mit längeren Teleskopen lassen sich höhere Vergrößerungen erzielen als mit kürzeren. Schon bei den ersten Fernrohren ließ sich der Abstand zwischen Okular und Objektiv verändern. Dies geschah, um Objekte in 
verschiedenen Entfernungen scharf stellen zu können, und auch, um evtl. vorhandene Refraktionsfehler des Beobachters, also Kurz- oder Weitsichtigkeit, ausgleichen zu können.

\section{Teleskopisches Sehen ohne Teleskop}

Akkommodation, die natürliche

Nahsicht junger Menschen

Ein junger Mensch kann akkommodieren, je jünger, umso besser. $U m 20 \mathrm{~cm}$ vor dem Auge scharf zu sehen, muss $5 \mathrm{dpt}$ akkommodiert werden, für eine Entfernung von $10 \mathrm{~cm}$ sind schon $10 \mathrm{dpt}$ zu akkommodieren. Ein Kind kann noch $14 \mathrm{dpt}$ akkommodieren, also in $7 \mathrm{~cm}$ scharf sehen. Die Akkommodation kann aber auch zum teleskopischen Sehen in der Ferne genutzt werden. Wenn von einem Fernrohr die Objektivlinse ausgebaut und frei vor das Auge gehalten wird, dann kann die Akkommodation die optische Wirkung des Okulars ersetzen. Wenn ein junger Mensch eine +1-dpt-Linse als Objektiv etwa $1,1 \mathrm{~m}$ vor sein Auge hält und $10 \mathrm{dpt}$ akkommodiert, sieht er durch die Linse Entferntes 10-fach vergrößert, aber auf dem Kopf stehend (@ Tab.2). Die Werte der ersten 4 Zeilen von $\bigcirc$ Tab. 1 sind also nicht nur mit einem Okular, sondern auch durch Akkommodation zu erzielen. Die Qualität des Bildes kann dem eines Bildes mit einem einlinsigen Okular überlegen sein.

Eine Akkommodation von $10 \mathrm{dpt}$ ist spontan schwer zu erzeugen. Wenn ein junger Mensch jedoch einen Finger als Akkommodationsreiz $10 \mathrm{~cm}$ vor das Auge in Richtung einer Objektivlinse hält, erkennt er in der Objektivlinse das vergrößerte Bild von entfernten Gegenständen auf dem Kopf stehend gestochen scharf. Er wird merken, dass er die Vergrößerung stufenlos variieren kann: Durch verstärkte Akkommodation kann er das Abbild heranzoomen, wenn er seinen Kopf gleichzeitig ein wenig der Linse nähert. Die 3. Spalte in 0 Tab. 1 zeigt nicht nur die Länge eines Fernrohrs, sondern gleichzeitig auch die Entfernung, die das Auge für ein teleskopisches Sehen von der Objektivlinse entfernt sein muss.

Das teleskopische Sehen mit einer + 1dpt-Objektivlinse ist technisch einfach zu erzielen, wenn sie in Augenhöhe auf ein Fenster geklebt wird und der Horizont aus einer Entfernung von $110 \mathrm{~cm}$ mit $10 \mathrm{dpt}$ Akkommodation anvisiert wird. Einfacher ist es, wenn eine +2 -dpt-Linse in etwa $60 \mathrm{~cm}$ vor das Auge gehalten wird. Dann ist mit 10 dpt Akkommodation zwar nur eine Vergrößerung von 5fach zu erreichen ( $\bullet$ Tab. 1 und 2), aber das teleskopische Sehen ist überall und in alle Richtungen möglich.

\section{Kurzsichtigkeit (Myopie) und} Übersichtigkeit (Hyperopie)

Auch ein älterer, wenig akkommodationsfähiger Mensch kann durch eine Linse mit einer Brennweite von $50 \mathrm{~cm} \mathrm{(+2dpt)}$ noch eine Vergrößerung von 2,5-fach erreichen, wenn er $5 \mathrm{dpt}$ kurzsichtig (myop) ist und seine Brille ablegt (○ Tab.2). Der junge Kurzsichtige kann Myopie und Akkommodation kombinieren: Wenn ein 5dpt-Myoper noch $5 \mathrm{dpt}$ akkommodiert, kann er durch eine Objektivlinse mit einer Brennweite von $50 \mathrm{~cm}$ ( $2 \mathrm{dpt}$ ) eine 5-fache Vergrößerung erreichen, sobald er seine Brille oder Kontaktlinse ablegt. Die Abbildungen stehen aber auf dem Kopf.

Kepler hat 1611 das hier beschriebene Sehen eines „umgekehrten Bildes durch eine Konvexlinse" von Myopen im Lehrsatz 78 erwähnt, ohne auf die Sonderform der physiologischen Myopie durch Akkommodation einzugehen ([7], S. 480). Im Satz 28 berichtet er 1604 über seine eigene Myopie ([9], S. 273). Wahrscheinlich hat er das umgekehrte Bild nicht nur theoretisch errechnet, sondern auch praktisch ausprobiert, indem er ein Glas einer Lesebrille mit ausgestreckter Hand vor sich hielt. Da er nur schwach myop war und 1611, mit 39 Jahren, nur noch wenig akkommodieren konnte, hat er den möglichen Vergrößerungseffekt nicht selber erlebt und beschrieben.

Etwas ungünstiger ist es für den Übersichtigen. Wenn er akkommodiert, schwächt er seine Hyperopie ab. Ohne Akkommodation kann ein Hyperoper, der eine +5-dpt-Brille benötigt, durch eine 1-dpt-Objektivlinse eine 5-fache Vergrößerung erzielen. Dazu muss eine +1-dpt-Objektivlinse $80 \mathrm{~cm}$ vor das Auge gehalten werden. Das Bild steht richtig herum ( Tab. 1 und 2). Diese Methode hat Rolf Riekher beschrieben [4].

Ein alterssichtiger Mensch ohne Refraktionsfehler kann solche Versuche ebenso einfach durchführen, wenn er die Refraktion durch eine „falsche“ Brille ändert. Das teleskopische Sehen kann entsprechend der Werte von $\bullet$ Tab. 1 erzielt werden, wenn die Objektivlinse, wie oben beschrieben, frei vor das Auge gehalten wird und anstelle des Okulars eine Probierbril- le mit einseitigen Werten von beispielsweise +5 dpt oder $-5 \mathrm{dpt}$ aufgesetzt wird. Wenn wir heute die o.g. Versuche mit handelsüblichen Brillengläsern vom Optiker durchführen, werden wir enttäuscht sein, da die meniskenförmig gewölbten Brillengläser zum Einsatz in unmittelbarer Nähe des Auges hergestellt sind. Für ein teleskopisches Sehen verzerren die Gläser zu sehr. Die Versuche lassen sich einfacher durchführen, wenn von einem billigen Teleskop mit einem Objektivdurchmesser von mindestens $5 \mathrm{~cm}$ und einer Brennweite von mindestens $50 \mathrm{~cm}$ die Objektivlinse ausgebaut wird. Je größer der Objektivdurchmesser, umso größer ist bei niedriger Vergrößerung das Gesichtsfeld.

Einzelne mehr oder weniger genaue kasuistische Berichte über ein Vergrößerungsglas für die Ferne sind schon vor Kepler überliefert. Leonardo da Vinci (1452-1592) war wohl hyperop und hat die Methode angewendet [6]. Drei sehr lesenswerte und sich ergänzende Zusammenstellungen über die Vor- und Frühgeschichte des Fernrohrs geben Riekher, Willach und Rienitz; letzterer hat auch das teleskopische Sehen ohne Teleskop ausführlich berücksichtigt [4-6].

\section{Adam Elsheimer (1578-1610) $\nabla$}

Adam Elsheimer ist ein bedeutender deutscher Maler des 17. Jahrhunderts. Er wurde 1578 in Frankfurt geboren und starb 1610 in Rom. Rubens beklagte nach Elsheimers frühem Tod, dass Elsheimer in der Lage gewesen sei „Dinge, die keiner gesehen hat und keiner je sehen wird" darzustellen ([1], S.51). Von ihm sind heute noch etwa 30-40 Bilder erhalten, die auf Kupferbleche gemalt worden sind $[1,3,10]$. Die kleinsten sind $7 \times 9 \mathrm{~cm}$ groß Die „Flucht nach Ägypten“ ist mit $31 \times$ $41 \mathrm{~cm}$ eines der größten Bilder, das er gemalt hat. Er hatte eine große Sensibilität für optische Phänomene. In mehreren Bildern malte er die Vergrößerungen und Spiegelungen durch kugelige Gefäße ([3], S.151-153). Auch gab er die Parallaxe durch die Spiegelung des Mondes im Wasser in der "Flucht nach Ägypten“ richtig wieder: Der Mond ist in der Spiegelung näher an den Bäumen als am Himmel. Diese Komposition Elsheimers wurde von späteren großen Künstlern wie Rubens, mit dem er eng befreundet war, und Rembrandt übernommen und modifiziert, von vielen anderen Künstlern kopiert $[1,3]$. 


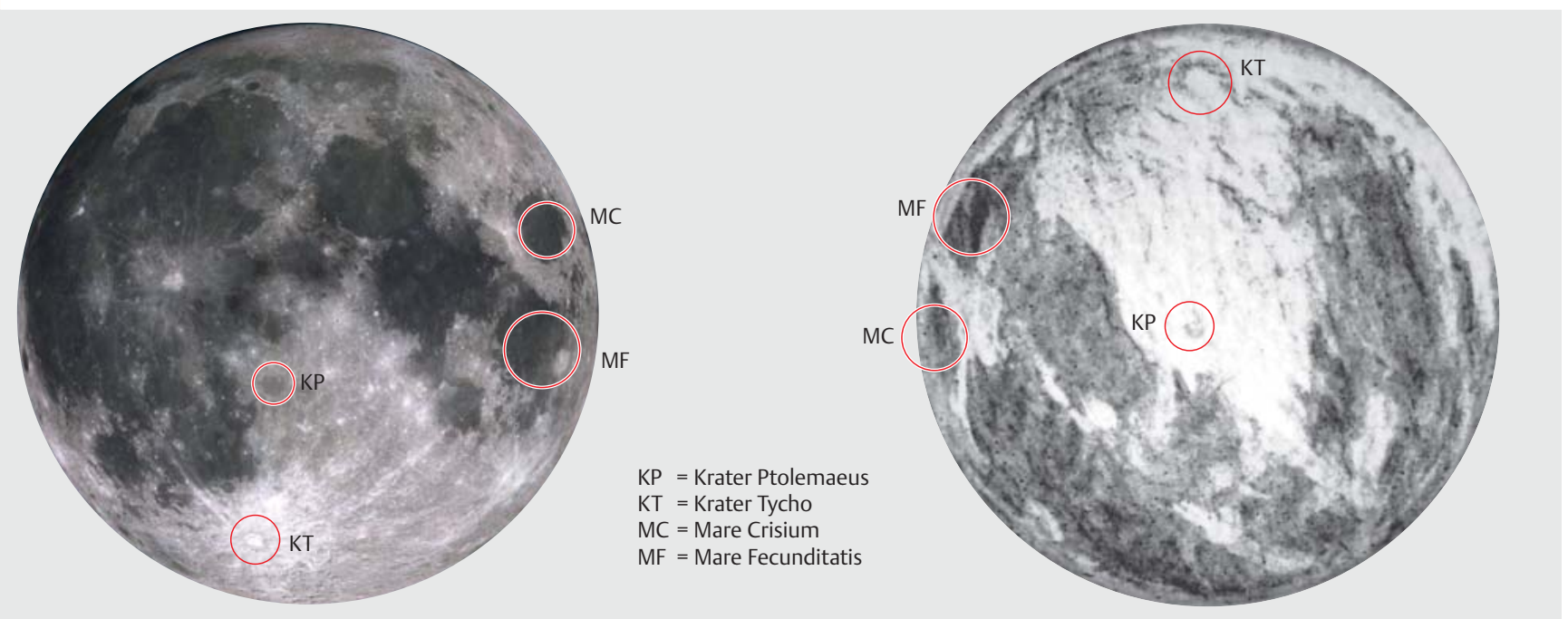

Abb.2 Links: Abbildung vom Mond, wie wir ihn als Vollmond am nächtlichen Himmel sehen: Die dunklen Flächen der "Mare“ sind vorwiegend oben. Rechts: Abbildung vom Mond, wie ihn Adam Elsheimer gemalt hat: Die dunklen Flächen der „Mare“ sind vorwiegend runten. Der Mond steht „auf dem Kopf“.

Adam Elsheimer arbeitete sehr langsam und bei verschiedenen Bildern ist nicht klar, ob er sie selber zu Lebzeiten noch vollenden konnte $[1,3]$. Auch die genaue Datierung seiner Bilder ist oft fraglich, er hat sie nicht signiert. In der „Flucht nach Ägypten“ fehlt noch die Spiegelung eines großen dürren Baumes direkt links vom Mond im Wasser. Auf der Rückseite des Bildes wurde nachträglich der Vermerk „Adam Elsheimer fecit 1609“ angebracht ([11], S. 24). Wahrscheinlich war es schon 1608 weitgehend fertiggestellt, als Rubens Rom verließ und Elsheimer das letzte Mal sah ([1], S. 51; [12], S. 191).

Zur Darstellung von astronomischen Details in seiner „Flucht nach Ägypten“ gibt es 3 Fragen, die bisher nicht übereinstimmend beantwortet werden konnten und die hier besonders interessieren: 1) Malte er mit Lupe? 2) Wie erkannte er die Sterne der Milchstraße? 3) Warum malte er den Mond auf dem Kopf?

\section{War eine Lupe für Elsheimer nötig?} Elsheimers Bilder enthalten derart feine Details, dass sie nach Meinung vieler mithilfe einer Lupe gemalt worden sein müssen ([1], S. 28; [3], S. 131, S. 151). In der Aufstellung des Inventars nach seinem Tod wird jedoch keine Lupe gefunden ([1], S. 48-49). Mit Sicherheit konnte Adam Elsheimer noch sehr gut akkommodieren, als er mit 22 Jahren nach Rom kam, und konnte ohne Lupe bis zu $10 \mathrm{~cm}$ in der Nähe sehen.

Darüber hinaus wäre denkbar, aber für unsere Überlegungen nicht notwendig, dass er im Laufe seiner Tätigkeit myop geworden ist. Schon Johannes Kepler hat vermutet ([7], S. 474), dass Naharbeit bei schlechter Beleuchtung, wie sie Adam Elsheimer ausgeübt hat, die Entwicklung einer Myopie fördert. Diese Theorie findet auch heute noch viele Befürworter [13-15]. Der spanische Maler Jusepe Martinez berichtete später über seinen deutschen Kollegen: „Er war sehr zurückgezogen und von beschaulicher Art, so sehr, dass er auf den Straßen wie abwesend ging, und dass er mit niemanden sprach, wenn man ihn nicht anredete“ ([10], S.39). Augenärzten ist die Klage von Patienten mit Sehstörungen aller Art sehr geläufig, dass sie ihre Bekannten auf der Straße nicht mehr erkennen können.

\section{Warum malte Elsheimer die Milch- straße aus über 1000 Sternen?}

Die detaillierte Darstellung des nächtlichen Himmels gilt als Novum in der Kunstgeschichte. Die Milchstraße ist zum ersten Mal als eine Ansammlung von 1500 Einzelsternen dargestellt ([16], S. 114). Wie konnte Elsheimer 1609 gewusst haben, dass die Milchstraße kein Nebel ist? Alle Autoren, die dieses Problem erkannten, vermuten, dass er zum Malen des Bildes auf ein Teleskop angewiesen war. 1609 besaß Federico Cesi in Rom bereits ein Teleskop nach dem Prinzip von Lippershey mit einem konkaven Okular und aufrechtem Bild. Das könnte er genutzt haben, so wurde bisher gemutmaßt ([3], S. 146; [16], S. 124). Hätte dann nicht Elsheimer dem Besitzer gleich seine Beobachtung gezeigt, und wäre es nicht wie ein Lauffeuer durch Rom ge- gangen? Bei der Anfertigung des Mondes und des Sternenhimmels konnte er auch nicht durch die ersten Publikationen astronomischer Beobachtungen des Himmels mit dem Teleskop in den Niederlanden (1608) oder 1610 in Italien beeinflusst worden sein ([16], S. 125).

Wahrscheinlich hat Elsheimer, der in sehr armen Verhältnissen lebte, nicht eines der extrem seltenen und sehr teuren Teleskope genutzt, sondern lediglich ein einfaches, schwaches Brillenglas. Mit diesem Glas wird er experimentiert haben, bis er das teleskopische Sehen entdeckt hat. Er war noch deutlich jünger als Johannes Kepler beim Schreiben seiner „Dioptrice“ (1611) und konnte demnach besser akkommodieren. So hat er nicht nur das umgekehrte Bild, sondern auch den Vergrößerungseffekt durch Akkommodation bemerken können. Vielleicht war er auch schon stärker myop als Kepler? Beide hatten einen gemeinsamen Freundeskreis ([10], S. 22). Im Gegensatz zu einem Teleskop reicht hier das Weitergeben der Linse zur Demonstration des Gesehenen nicht aus: Wenn ein Beobachter nicht sehr kurzsichtig ist oder stark akkommodiert, kann er durch die Linse nichts erkennen. Was bisher eher Hypothese ist, wird durch die Darstellung des Mondes zur wahrscheinlichsten Erklärung seines teleskopischen Sehens.

\section{Warum steht der Mond auf dem Kopf?}

Wenn die Details des Mondes genau betrachtet werden, so sehen wir nicht das uns bekannte „Mondgesicht“, sondern er 
steht auf dem Kopf ([17]; [18], S. 126)! Dabei hat Adam Elsheimer Krater und andere Details in den nur etwa $1 \mathrm{~cm}$ großen Mond gemalt, die wir im Bild nur mit Lupe und am freien Himmel nur mit Teleskopen erkennen können ( Abb. 2). Sowohl in der astronomischen, als auch in der kunstgeschichtlichen Fachliteratur wurde seit über 40 Jahren vielfach diskutiert, wie das möglich ist, zuletzt bei Thielemann (2014) und Bredekamp (2015). Eine befriedigende Antwort wurde von keinem gefunden. Die ersten Fernrohre mit konkaven Okularen, mit denen ab 1609 Thomas Harriot, Simon Marius, David Fabricius, Galileo Galilei und viele andere astronomische Beobachtungen begannen, lieferten immer ein aufrechtes Bild. Erst 1613, 3 Jahre nach Elsheimers Tod, gab es die moderneren Teleskope nach Kepler mit konvexer Okularlinse, die ein umgekehrtes Bild erzeugen! Nur das von Kepler allgemein und hier im Detail beschriebene teleskopische Sehen mit einer schwachen konvexen Linse als Objektiv sowie Akkommodation oder Myopie als Okularersatz liefert die wahrscheinliche Antwort, warum Elsheimer die Sterne der Milchstraße, Krater vom Mond sowie sein umgekehrtes Bild in der „Flucht nach Ägypten“ schon um 1608 erkennen und malen konnte. War es demnach ein Künstler und nicht ein Wissenschaftler, der uns erstmals die teleskopischen Geheimnisse des Universums nahe gebracht hat?

\section{Danksagung}

Herzlichen Dank an Herrn Prof. Dr. Wilhelm Seggewiß für die Beratung und Korrekturen in physikalischen und astronomischen Details.
Interessenkonflikt: Nein.

\section{Wenzel}

Augenklinik Petrisberg, Trier

\section{Literatur}

1 Andrews K. Adam Elsheimer. Gemälde, Zeichnungen und Radierungen. München: Schirmer/Mosel; 2006

2 Baumstark R, Hrsg. Von neuen Sternen. Adam Elsheimers Flucht nach Ägypten. München: Pinakothek-DuMont; 2005

3 Thielemann A. Natur Pur? Literarische Quellen und Philosophische Ziele der Naturdarstellungen bei Adam Elsheimer. In: Thielemann A, Gronert S, Hrsg. Adam Elsheimer in Rom. München: Werk-Kontext-Wirkung Hirmer; 2008: 125-156

4 Riekher R, Beck HG. Fernrohre und ihre Meister. Jena: Astrobeck; 1990: 11-50

5 Willach $R$. Der lange Weg zur Erfindung des Fernrohres. In: Hamel J, Keil I, Hrsg. Der Meister und die Fernrohre. Frankfurt: Acta Historica Astronomiae 33; 2007: 34-126

6 Rienitz J. Historisch-physikalische Entwicklungslinien optischer Instrumente. Lengerich: Pabst; 1999: 66-139

7 Kepler J. Dioptrice (1611; übersetzt von F. Plehn). In: Riekher R, Hrsg. Schriften zur Optik. Frankfurt: Wissenschaftlicher Verlag Deutsch; 2008: 441-526

8 Daxecker F. Christoph Scheiner und die Optik des Auges. Spektrum Augenheilkd 2004; 18: 201-204

9 Kepler J. Ad Vitellionem paralipomena (1604; übersetzt von F. Plehn). In: Riekher R, Hrsg. Schriften zur Optik. Frankfurt: Wissenschaftlicher Verlag Deutsch; 2008: 73296

10 Klessmann R. Im Detail die Welt entdecken. Adam Elsheimer 1578-1610. Städelsches Kunstinstitut Frankfurt: Edition Minerva; 2006

11 Deckiert M. Ein Werk, das in allen Theilen zugleich und in einem jeden besonerlich ganz unvergleichlich ist. Adam Elsheimers Flucht nach Ägypten - Werk und Wirkung. In: Baumstark R, Hrsg. Von neuen Sternen. Adam Elsheimers Flucht nach Ägypten. München: Pinakothek-DuMont; 2005: 24

12 Klessmann R. Fragen zur Ausbreitung und Wirkung der Werke Elsheimers. In: Baumstark R, Hrsg. Von neuen Sternen. Adam Els- heimers Flucht nach Ägypten. München: Pinakothek-DuMont; 2005: 179-196

13 Schaeffel F. Das Rätsel der Myopie. Störungen in der Feinabstimmung von Länge und Brennweite des Auges. Ophthalmologe 2002; 99: 120-141

14 Dolgin E. Myopie. Im Internet: www. spektrum.de/news/brille-gefaellig-stubenhocker/1341152; Stand: 29.6.2015

15 Deutsche Ophthalmologische Gesellschaft (DOG). Viel Licht setzt Neurotransmitter Dopamin in der Netzhaut frei - Spielen im Freien schützt Kinder vor Kurzsichtigkeit. Pressemitteilung vom 29. 4. 2015. Im Internet: $\quad$ www.dog.org/?cat $=224$; $\quad$ Stand: 29.6.2015

16 Hartl G, Sicka C. Komposition oder Abbild? Die Darstellung des Nachthimmels in Adam Elsheimers Flucht nach Ägypten - eine naturwissenschaftlich-kritische Betrachtung. In: Baumstark R, Hrsg. Von neuen Sternen. Adam Elsheimers Flucht nach Ägypten. München: Pinakothek-DuMont; 2005: 107-125

17 Hartl G. Das Universum des Adam Elsheimer. Astronomie heute 3/2007 S. 16 und 5/ 2007 S. 7

18 Thielemann A. Himmelsfeuer: Kosmologie und Theologie in Adam Elsheimers „Flucht nach Ägypten“. In: von Albrecht A, Cordibella G, Hrsg. Tintenfass und Teleskop. Galileo Galilei im Schnittpunkt wissenschaftlicher, literarischer und visueller Kulturen im 17. Jahrhundert. Berlin: W. de Gruyter 2014: 126-130

19 Bredekamp H. Galileis Denkende Hand. Form und Forschung um 1600. Berlin: W. de Gruyter; 2015: 99-100

\section{Bibliografie}

DOI http://dx.doi.org/10.1055/s-0035-1557827 Klin Monatsbl Augenheilkd 2015; 232: 1105-1109 (c) Georg Thieme Verlag KG Stuttgart · New York · ISSN 0023-2165

\section{Korrespondenzadresse}

Prof. Martin Wenzel

Augenklinik Petrisberg

Max-Planck-Str. 14-16

54296 Trier

Tel.: + 49/(0)651/9984990

Fax: $+49 /(0) 651 / 99849919$

wenzel-trier@gmx.de 